\title{
B10 - COMPARAÇÃO DE EFICÁCIA E SEGURANÇA DA ALFAEPOETINA DE BIO-MANGUINHOS COM UMA ALFAEPOETINA BIOSSIMILAR: ENSAIO CLÍNICO RANDOMIZADO
}

$\underline{\text { Paulo D. Picon }}^{1,2,3}$, Suzane Cristina Milech Pribbernow ${ }^{2}$, Carlos Alberto Prompt ${ }^{1,2}$, Amanda Quevedo ${ }^{2}$, Veronica Verleine Horbe Antunes ${ }^{2}$, Bianca Paula Mentz ${ }^{2}$, Fernando Comunello Schacher ${ }^{2}$

Instituições: 1-UFRGS, 2-HCPA, 3-SES-RS

Introdução: A anemia é uma complicação comum em pacientes com doença renal crônica (DRC) em hemodiálise e decorre principalmente da deficiência de eritropoetina. No Brasil, o tratamento com alfaepoetina é oferecido gratuitamente pelo Sistema Único de Saúde e utilizado por cerca de $90 \%$ da população de pacientes em tratamento com hemodiálise. Desde o segundo semestre do ano de 2006 o Laboratório Bio-Manguinhos (FioCruz, Ministério da Saúde) produz a alfaepoetina que vem sendo dispensada, no Brasil, aos pacientes portadores de DRC.

Objetivo: avaliar a eficácia e segurança da alfaepoetina produzida pelo laboratório BioManguinhos em relação a uma biosimilar anteriormente dispensada pelas secretarias estaduais e municipais de saúde. Material e Métodos: ensaio clínico, randomizado e duplo-cego em pacientes adultos em hemodiálise, alocados para dois grupos que receberam ou alfaepoetina produzida por Biomanguinhos-FioCruz ou alfaepoetina Blaussigel® por um período de seis meses, assumindo o pressuposto da equivalência entre ambos os produtos. Todos os pacientes foram avaliados mensalmente pelos médicos participantes do estudo, os resultados dos seus exames revisados e a dose de alfaepoetina ajustada com o objetivo de manter a hemoglobina entre 11 e 12 g\%. Segurança foi avaliada por eventos adversos e perfil laboratorial. O desfecho de interesse foi o nível de hemoglobina e sua variação ao longo do tempo nos dois grupos.

Resultados: 86 pacientes foram triados, tendo sido 74 randomizados, 36 e 38 , respectivamente nos grupos que receberam alfaepoetina Bio-Manguinhos (EPO BioManguinhos) e alfaepoetina biosimilar (EPO-Biosimilar). No baseline e nos 6 meses do estudo os dois grupos experimentais apresentaram similaridade em todos os parâmetros estudados (níveis séricos de hemoglobina, ferritina, ferro, índice de redução de uréia 
(URR) e hormônio paratireoideo, dose de epoetina UI/kg, potássio sérico, proporção de diabete melito). A taxa de eventos adversos foi semelhante nos 2 grupos.

Conclusões: A alfaepoetina humana recombinante produzida pelo Instituto de Tecnologia em Imunobiológicos da Fundação Oswaldo Cruz (BioManguinhos/Fiocruz/MS) mostrou eficácia e segurança similar à da Blausigel, em pacientes com insuficiência renal crônica em hemodiálise. 